\title{
Analysis and Design of a Metamaterial Lens Antenna Using the Theory of Characteristic Modes
}

\author{
Daniel Santillán-Haro, ${ }^{1,2}$ Eva Antonino-Daviu (D), ${ }^{1}$ Daniel Sánchez-Escuderos, ${ }^{1}$ \\ and Miguel Ferrando-Bataller $\mathbb{B}^{1}$ \\ ${ }^{1}$ Instituto de Telecomunicaciones y Aplicaciones Multimedia (ITEAM), Universitat Politècnica de València (UPV), c/Cami de Vera, \\ s/n, 46022 Valencia, Spain \\ ${ }^{2}$ Facultad de Ingeniería, Universidad Nacional de Chimborazo (UNACH), Avda. Antonio Jose de Sucre km 1.5 vía a Guano, \\ Riobamba, Ecuador
}

Correspondence should be addressed to Eva Antonino-Daviu; evanda@upvnet.upv.es

Received 29 September 2017; Accepted 18 December 2017; Published 18 February 2018

Academic Editor: Safieddin Safavi-Naeini

Copyright (c) 2018 Daniel Santillán-Haro et al. This is an open access article distributed under the Creative Commons Attribution License, which permits unrestricted use, distribution, and reproduction in any medium, provided the original work is properly cited.

\begin{abstract}
A new single-layer metamaterial lens antenna aimed to operate at $10 \mathrm{GHz}$ is proposed in this paper. The lens antenna consists of twelve capacitively coupled unit cells distributed along a ring and illuminated by an open-ended circular waveguide with a metallic resonant ring. The theory of characteristic modes is used to analyze the metamaterial lens, in order to provide an insight into the radiation characteristics of the antenna. The proposed antenna has been optimized, obtaining a large bandwidth and a maximum directivity of $12.88 \mathrm{dBi}$ at $10 \mathrm{GHz}$.
\end{abstract}

\section{Introduction}

Metal lenses have attracted an increasing interest during the last decade due to their multiple applications [1-9]. In this context, different lenses have been recently proposed aimed at maximizing the gain and the efficiency of the radiating structures. These lenses are formed by periodic structures, for example, EBG (electromagnetic band gap) or FSS (frequency selective surfaces), which provide the aforementioned characteristics in a low-profile shape. In $[1,2]$, a type of FSS, the so-called metallic hole array (MHA), was used to design a low-profile planar lens at millimeter-wave frequencies, while in [3], an extended version was proposed, which was fed by two open-ended waveguides.

Periodic structures are also used to design transmitarrays $[4,5]$ and reflectarrays $[6]$ by means of an intensive study of the physical model of the feed element and the unit cell. Additionally, the compactness of metamaterial lens antennas makes them very attractive for the low-cost development of metal lenses at terahertz frequencies [7], thin planar lenses for massive MIMO applications in the millimeter-wave band [8], or ultrathin planar lens antennas based on gradient metasurfaces [9].

So far, the design of the proposed metal lenses has been based on the analysis of the unit cell with infinitely periodic boundary conditions. However, the truncation of the lenses in a finite number of elements leads to inaccurate results. An alternative to this analysis consists of using the theory of characteristic modes (TCM). This theory was initially formulated by Garbacz and Turpin [10] and then refined by Harrington and Mautz $[11,12]$. The TCM was shown to be really helpful for the analysis and design of several antenna structures [13]. The popularity of this theory relies on the fact that it provides a very clear understanding of the radiating behavior of metallic and dielectric bodies, which ease the design process. In [11], an extensive explanation about the TCM and how to compute the characteristic eigencurrents and eigenvalues for a conducting body can be found.

In addition to the first applications of the TCM reviewed in [13], this theory has been recently used to analyze the bandwidth of a $2 \times 2$ bowtie array with a metasurface [14], design electrically small unmanned aerial vehicle (UAV) 


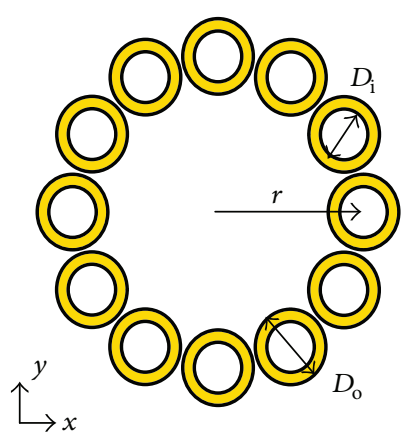

(a)

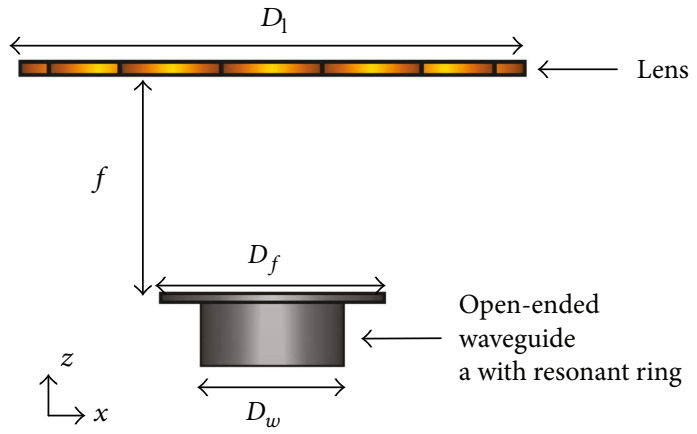

(b)

Figure 1: Geometry of the proposed lens antenna: (a) top view and (b) lateral view.

antennas [15], design and analyze slotted planar structures [16], or study some canonical structures such as spheres or cylinders [17]. Moreover, a single-layer metal lens formed by a central circular metallic ring surrounded by a set of eight metallic rings was analyzed using the TCM in [18], a wideband low-profile metasurface antenna was modeled and optimized using characteristic modes in [19], and a low-profile lens using metallic cylinders was investigated in [20] using the TCM.

This paper presents a new low-profile metallic lens antenna formed by twelve metallic rings distributed in a single layer and arranged along a ring, as illustrated in Figure 1. The structure is analyzed using the TCM, by means of the commercial electromagnetic simulators CST [21] and FEKO [22], in order to provide some physical insight into the radiating characteristics of the antenna.

The paper is organized as follows: Section 2 presents the geometry of the proposed antenna and the design procedure, Section 3 describes the characteristic modes of the metallic structure and the radiating behavior associated with the antenna, Section 4 shows the main results obtained with the design proposed at $10 \mathrm{GHz}$, and, finally, Section 5 highlights the main conclusions.

\section{Antenna Structure}

The complete system, formed by a feeding circular waveguide and a single-layer lens antenna, is shown in Figure 1(b). As can be observed, the feeding element is a circular aperture with a resonant ring, placed at a distance $f$ from the single-layer lens in the $z$-axis. The metallic ring attached to the circular aperture is used to maximize the gain and minimize the crosspolar level of the feeder. By optimizing the structure for the frequency band of interest $(9-11.5 \mathrm{GHz})$, the resulting dimensions are the following: $t=1.27 \mathrm{~mm}, D_{w}=19.35 \mathrm{~mm}$, and $D_{f}=33.89 \mathrm{~mm}$, where $t$ is the thickness of the metallic ring and $D_{w}$ and $D_{f}$ are the internal and external diameter, respectively.

Considering the previous feeder, the lens has been optimized to maximize the gain and minimize the sidelobe level and the crosspolar level of the antenna. As a result, the following dimensions have been obtained: $D_{\mathrm{i}}=$ $10.88 \mathrm{~mm}, D_{\mathrm{o}}=15.58 \mathrm{~mm}, r=30.55 \mathrm{~mm}, D_{1}=76.68 \mathrm{~mm}$,

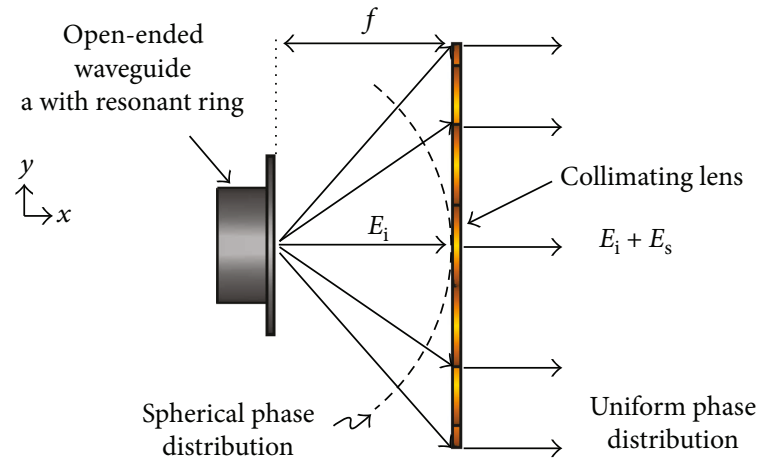

FIGURE 2: Schematic view of the proposed single layer lens antenna.

and $f=13.85 \mathrm{~mm}$, where $D_{\mathrm{i}}$ and $D_{\mathrm{o}}$ are the internal and external diameter, respectively, of the metallic ring, $r$ is the radius of the ring where the twelve unit cells are distributed, $D_{1}$ is the diameter of the lens, and $f$ is the focal length.

In order to improve the performance of the feeding aperture, the spherical phase distribution of the field on the lens is transformed into a uniform phase distribution above the lens, as indicated in Figure 2. Although the aforementioned optimization is performed in transmission, the lens can also be analyzed in reception to study the focusing effects of the lens. To do so, the metallic lens is illuminated by a plane wave using the full-wave electromagnetic tool CST [21].

The total field at a point in space is the sum of the incident field $\vec{E}_{\mathrm{i}}$ produced by the primary feeder and the scattered fields $\vec{E}_{\mathrm{s}}$ produced by the rings. The induced currents in the rings depend on the incident field $\vec{E}_{\mathrm{i}}$ and will be analyzed using the TCM in Section 3. If an isolated metallic ring is illuminated by a plane wave (see Figure 3(a)), it can be observed that, in the central part of the ring, the waves are not transmitted, which is equivalent to a cancellation of the incident waves with the fields near the induced currents. Nevertheless, if the complete lens consisting of twelve coupled rings is illuminated, as shown in Figure 3(b), there are more intense fields in the focal area, also produced by the sum of the incident plane wave and the near fields of the metallic structure.

The behavior described is caused by the induced currents in the rings, which depend on the incident field and on the equivalent impedance of the rings. These currents produce 


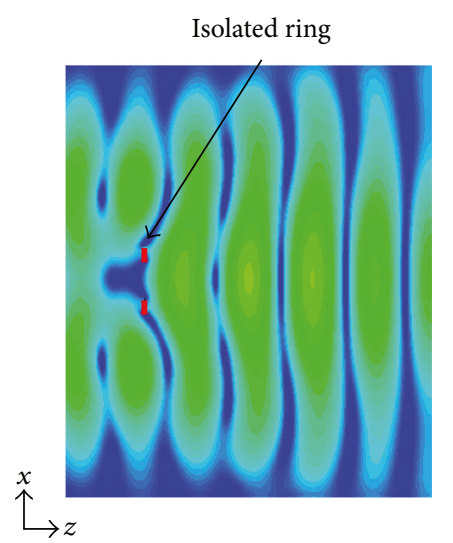

(a)

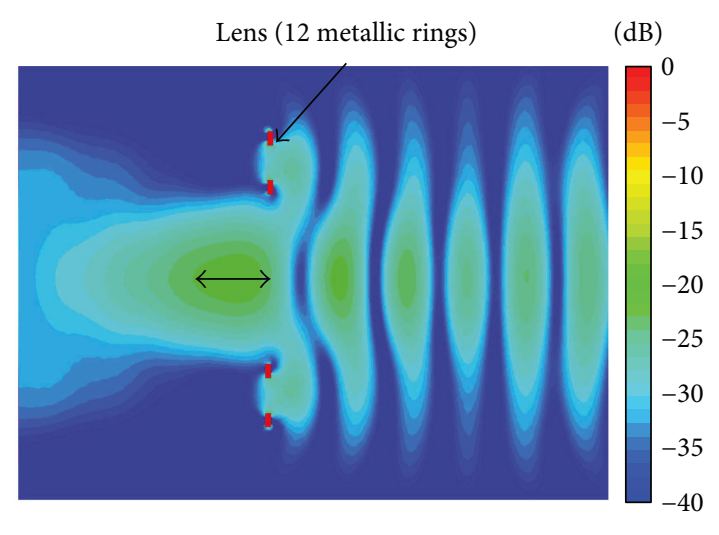

(b)

Figure 3: Simulation of an incident plane wave at $10 \mathrm{GHz}$ in (a) a metallic ring (unit cell) and (b) the proposed lens antenna. Color scale is valid for both plots.

a diffracted field throughout the space, which is added to the incident field. At the broadside, the incident field and the diffracted field are in phase; that is, the phases of the waves are compensated with the phase of the impedance of the metallic ring, and, consequently, the radiation pattern is maximized at this direction. Note that the design in transmission already takes into account all these effects.

From Figure 3(b), the focal distance $(f=13.85 \mathrm{~mm})$ can be deduced. This distance coincides with the focal distance at which the feeder was located for the optimization of the lens in transmission. Such focal distance, along with the diameter of the complete lens $\left(D_{1}=76.68 \mathrm{~mm}\right)$, confers the antenna $f / D_{1}$ ratio of 0.18 .

\section{Analysis of Characteristic Modes}

As exposed in $[10,11]$ and [13], the characteristic modes $\left(J_{n}\right)$ are real current modes that are extracted at every frequency from the generalized impedance matrix of the antenna. These characteristic modes form a set of orthogonal functions that can be used to expand the total current in the surface of the antenna, as described in the following equations:

$$
\begin{array}{r}
J=\sum_{n} \frac{V_{n}^{i} J_{n}}{1+j \lambda_{n}}, \\
V_{n}^{i}=\oint_{s} J_{n} \cdot E^{i} d s,
\end{array}
$$

where $J_{n}$ are the characteristic currents on the conducting body, $\lambda_{n}$ are the eigenvalues, and $V_{n}^{i}$ is the modal excitation coefficient expressed in (2). Associated with each eigenvalue, a characteristic angle can be defined as

$$
\alpha_{n}=180^{\circ}-\arctan \left(\lambda_{n}\right) .
$$

Observe that modes are capacitive for $\alpha_{n}>180^{\circ}$, inductive for $\alpha_{n}<180^{\circ}$, and resonate for $\alpha_{n}=180^{\circ}$ [13].

Nowadays, characteristic modes can be calculated using commercial electromagnetic simulators, such as CST [21] or FEKO [22]. In the next subsection, these simulators will be used to extract the characteristic modes associated with the proposed lens in order to evaluate the additional physical insight that the TCM brings into the radiation behavior of the antenna.

3.1. Characteristic Mode Analysis of the Proposed Planar Lens Antenna. In order to reveal the operation mechanism of the proposed lens antenna, the analysis of the modal behavior of the twelve metallic rings that form the antenna is investigated. The first four characteristic modes $\left(J_{n}\right)$ in the set of twelve metallic rings are plotted in Figure 4. As can be seen, currents of mode 1 form a loop inside and outside the total structure. modes 2 and 3 are a pair of degenerated modes [13], representing the modal current of the dominant modes, which generate a broadside radiation pattern. These broadside modes are the desired modes for the antenna design. Finally, mode 4 is a high-order mode. Radiation patterns associated with the first four modes of the proposed lens are shown in Figure 5.

The characteristic angle of the fundamental mode (mode 2 for vertical polarization) is shown in Figure 6(a). It is observed that the phase is about $160^{\circ}$ at $10 \mathrm{GHz}$, and the variation is smaller than $14^{\circ}$ for frequencies between $9 \mathrm{GHz}$ and $11.5 \mathrm{GHz}$, which allows a design with a large bandwidth. Figure 6(b) shows the modal significance, where it is observed that the 4 modes have similar values so it is necessary to analyze the contribution of each mode to the total radiated power of the proposed lens antenna for a plane wave illumination with vertical polarization. The results are shown in Figure 6(c), where mode 2 is the desired mode for broadside radiation.

As it has been discussed in the previous paragraph, the characteristic angle of the metallic ring indicates an inductive behavior involving a phase shift, as shown in Figure 6(a). In the proposed antenna, the unit cells receive a wave with a nonuniform phase distribution that must be compensated.

The two modes (mode 2 and mode 3 ) will lead to a linearly polarized antenna and facilitate the understanding of the modal behavior. By doing so, the characteristic mode analysis provides insightful guidelines for the excitation and implementation of the lens antenna. 


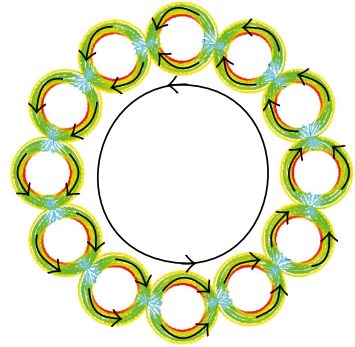

(a)

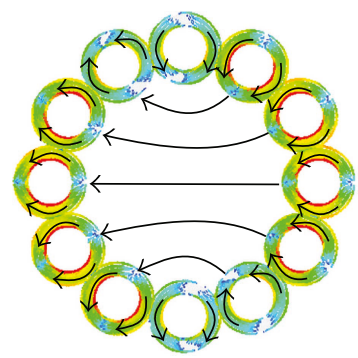

(c)

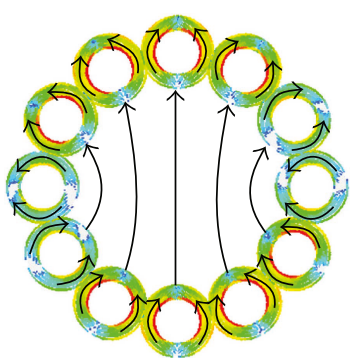

(b)

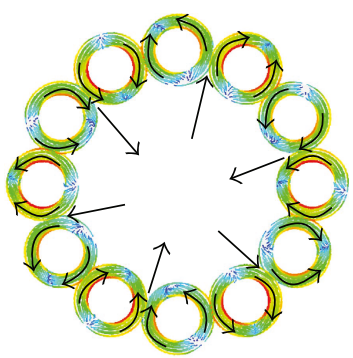

(d)

Figure 4: Modal currents of the proposed lens on the XY plane at $10 \mathrm{GHz}$ : (a) mode 1, (b) mode 2, (c) mode 3, and (d) mode 4.

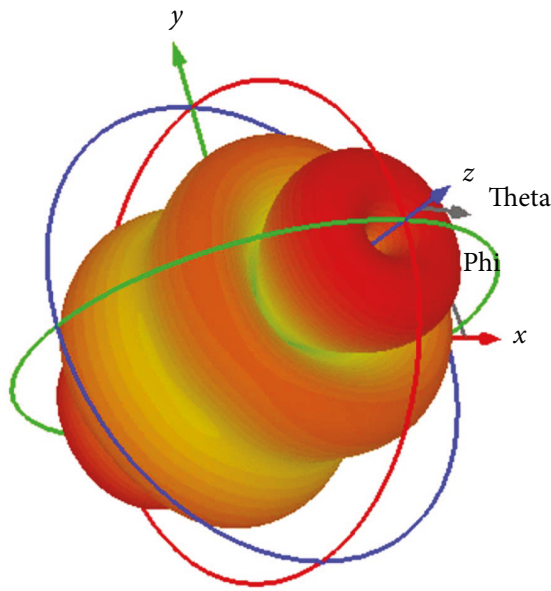

(a)

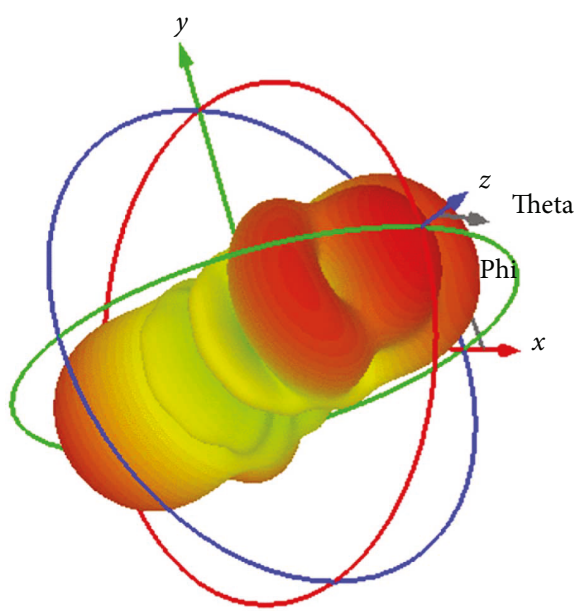

(c)

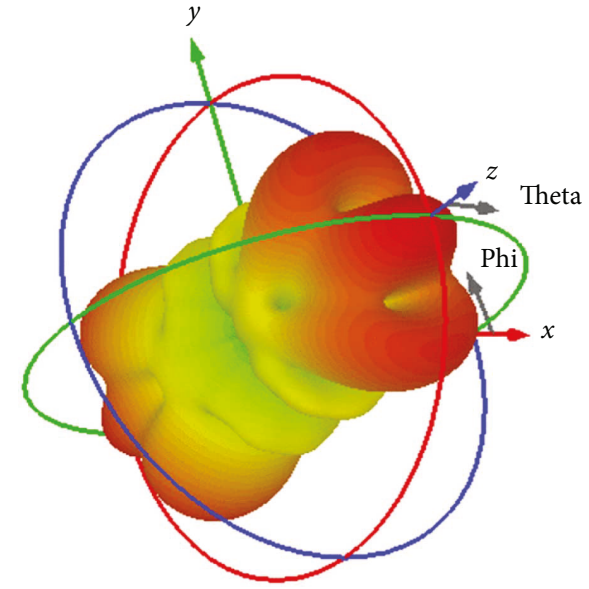

(b)

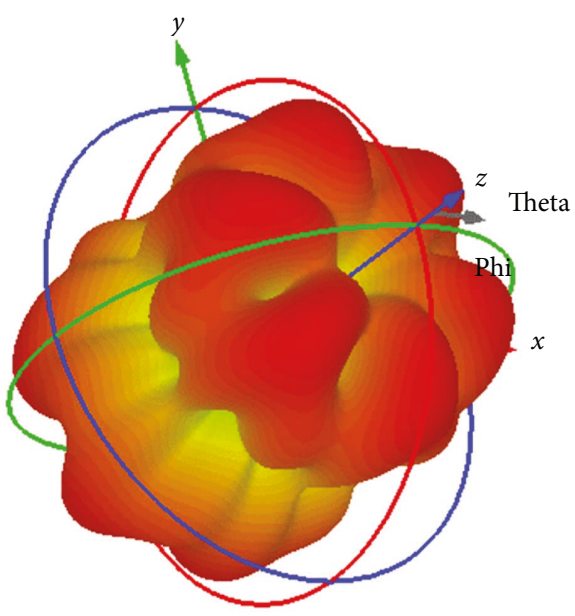

(d)

Figure 5: Modal radiation pattern of the proposed lens at $10 \mathrm{GHz}$. (a) mode 1, (b) mode 2, (c) mode 3, and (d) mode 4. 

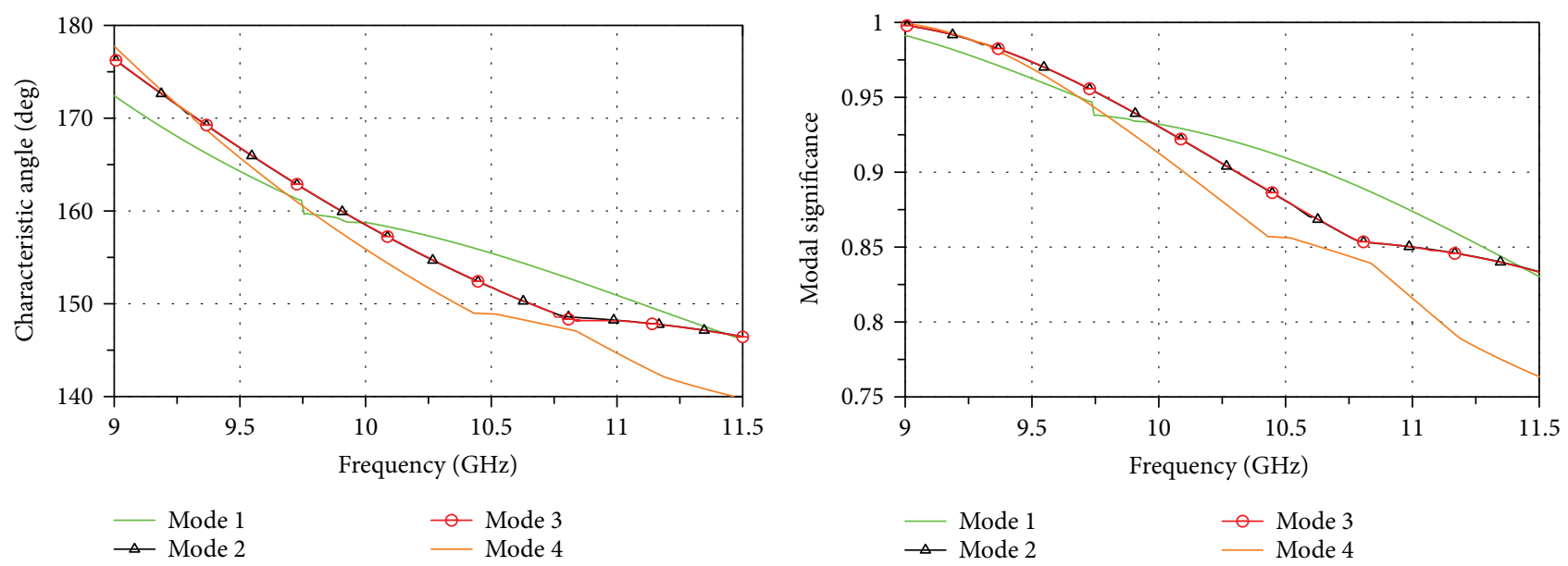

(a)

(b)

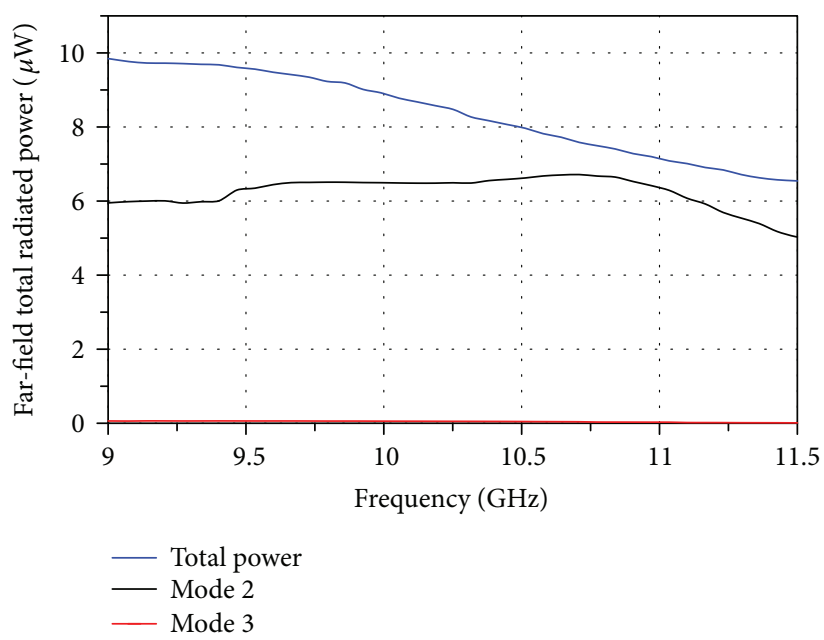

(c)

Figure 6: Modal parameters of the proposed lens: (a) characteristic angle, (b) modal significance, and (c) contribution of each mode to the total radiated power (including also the power of high-order modes).

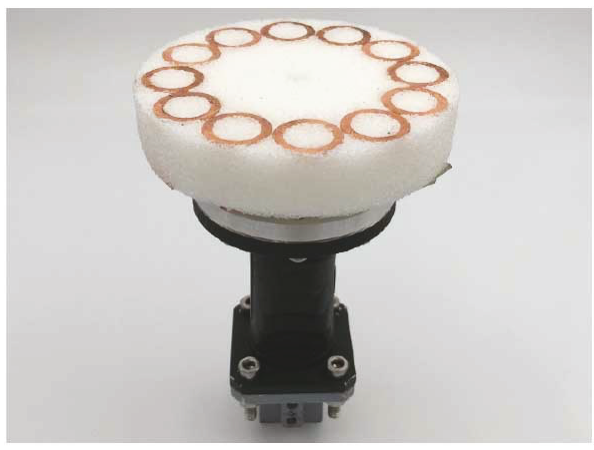

(a)

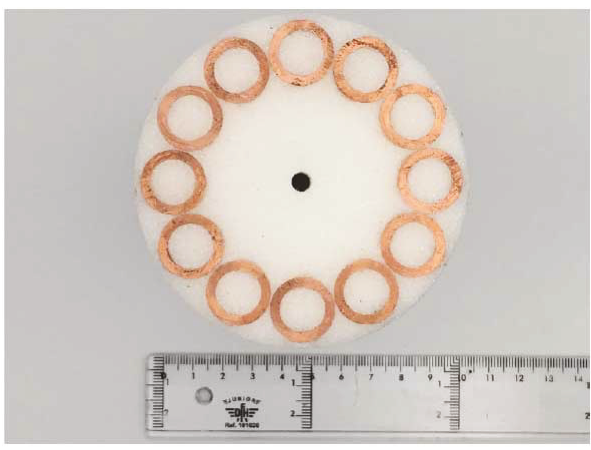

(b)

Figure 7: Pictures of the fabricated prototype: (a) complete structure and (b) lens.

3.2. Metamaterial Lens Antenna with Feeding Structure. In the proposed design, aimed to operate at $10 \mathrm{GHz}$, a rectangular to circular waveguide transition (model 1764 of FLANN) has been used to allow the feeding of the structure by means of a standard rectangular waveguide flange. Figure 7 shows two pictures of the fabricated prototype. The feeder (circular waveguide aperture with a resonant ring) has been fabricated in aluminum with a milling machine and has been attached to the transition. The lens' rings have been fabricated in a copper sheet and attached to a foam layer with the same 


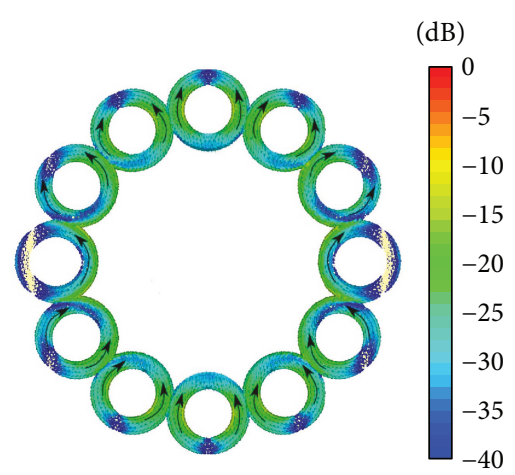

FIGURE 8: Surface current of the proposed lens antenna at $10 \mathrm{GHz}$.

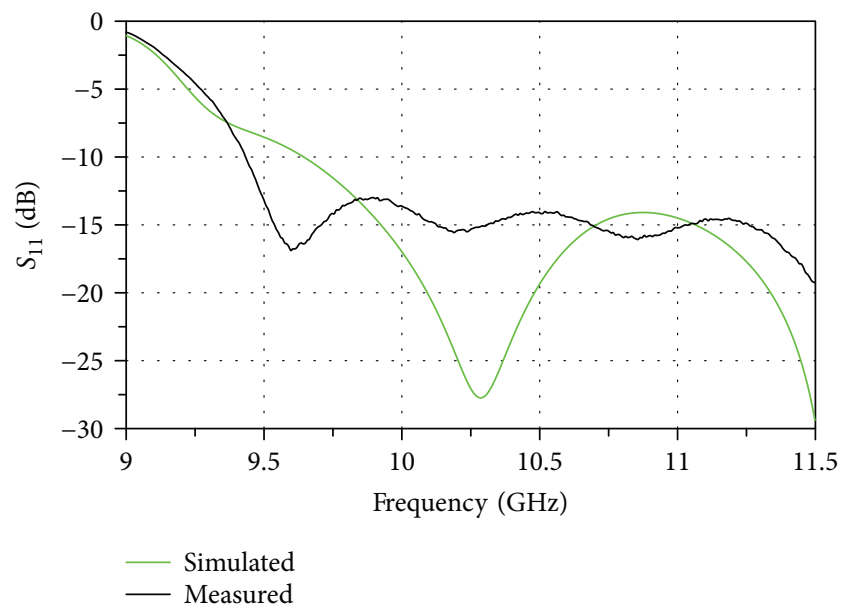

FIgURE 9: Measured $S_{11}$ parameter of the proposed lens antenna.

height as the focal distance to guarantee the correct separation between the lens and the feeder.

The total surface current in the proposed lens antenna at $10 \mathrm{GHz}$ is illustrated in Figure 8. As can be observed, the current distribution is similar to the one of dominant mode 2 (see Figure 4(b)). This mode has the same polarization as the incident wave and, consequently, provides the greatest contribution of power, as shown in Figure 6(c) (the total power includes the power of modes two and three, shown in Figure 6(c), and the power of higher-order modes).

\section{Results}

Figure 9 shows the measured $S_{11}$ parameter of the fabricated lens antenna. As can be observed, measured and simulated results are quite similar, with a good matching $\left(S_{11} \leq 10 \mathrm{~dB}\right)$ starting at $9.5 \mathrm{GHz}$, approximately, in both cases. The level of the $S_{11}$ is kept below $-10 \mathrm{~dB}$ up to $11.5 \mathrm{GHz}$. The response might have been extended beyond this upper frequency limit, but, as it will be seen later, the gain decays at $11 \mathrm{GHz}$ and, hence, it does not worth to consider a larger bandwidth.

Figures 10 and 11 show the radiation pattern on the $H$ plane and E-planes, respectively, at $10 \mathrm{GHz}$. The simulation of the crosspolar component is not included in the graph because the results of the simulator are ideal values. The axial

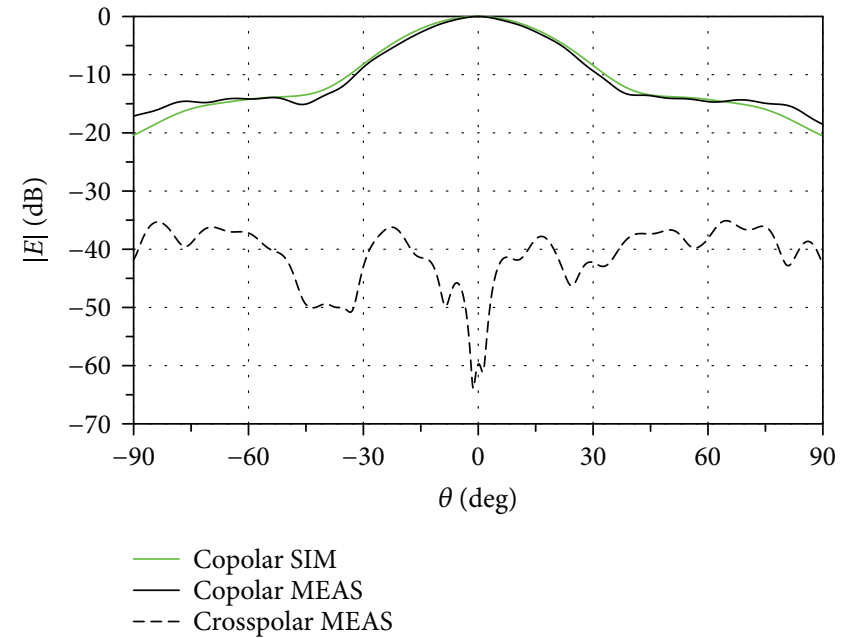

FIGURE 10: Normalized electric field radiation pattern in the $H$ plane of the proposed lens antenna at $10 \mathrm{GHz}$ : simulated (SIM) and measured (MEAS) copolar and crosspolar components.

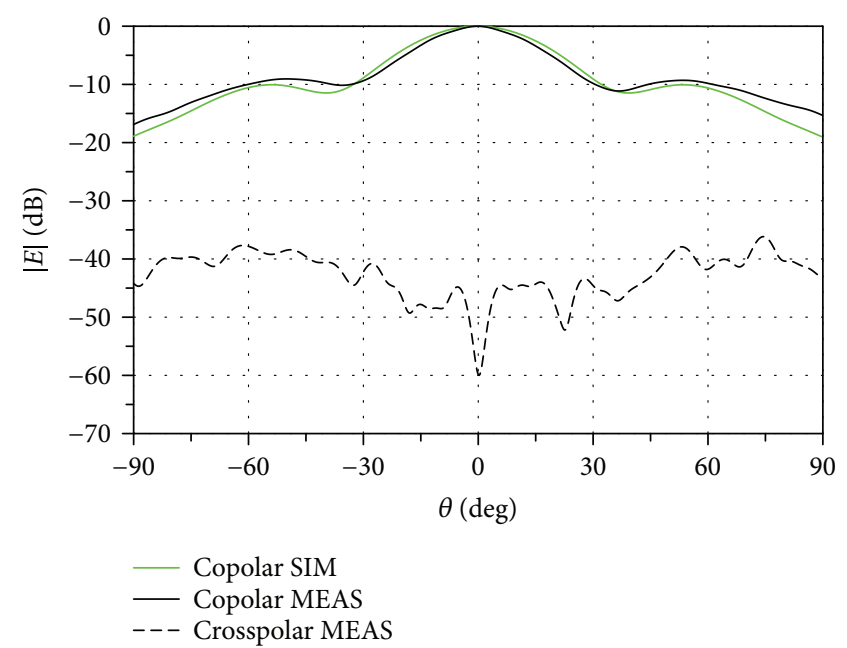

FIgURE 11: Normalized electric field radiation pattern in the E-plane of the proposed lens antenna at $10 \mathrm{GHz}$ : simulated (SIM) and measured (MEAS) copolar and crosspolar components.

symmetry of the radiation pattern (both planes present the same $-3 \mathrm{~dB}$ beamwidth and the same main beam shape) guarantees a good crosspolar level, below $-35 \mathrm{~dB}$ within the main beam.

Figure 12 shows the maximum gain of the lens antenna at different frequencies. As can be observed, the maximum gain is above $11.8 \mathrm{dBi}$ and the radiation efficiency is above $90 \%$ from $9.5 \mathrm{GHz}$ to $11.5 \mathrm{GHz}$. This high efficiency is caused by the use of low-loss materials in the manufactured prototype. Observe the aforementioned decay of the maximum gain from 11 to $11.5 \mathrm{GHz}$. At these frequencies, the rings are far from resonance and, consequently, the performance worsens.

The obtained maximum directivity is $12.88 \mathrm{dBi}$ at $10 \mathrm{GHz}$, which corresponds to a directivity increase of $5.71 \mathrm{~dB}$ with respect to an open-ended waveguide with the same aperture size as the feeder of the proposed lens antenna, as can be observed in Figure 13. 


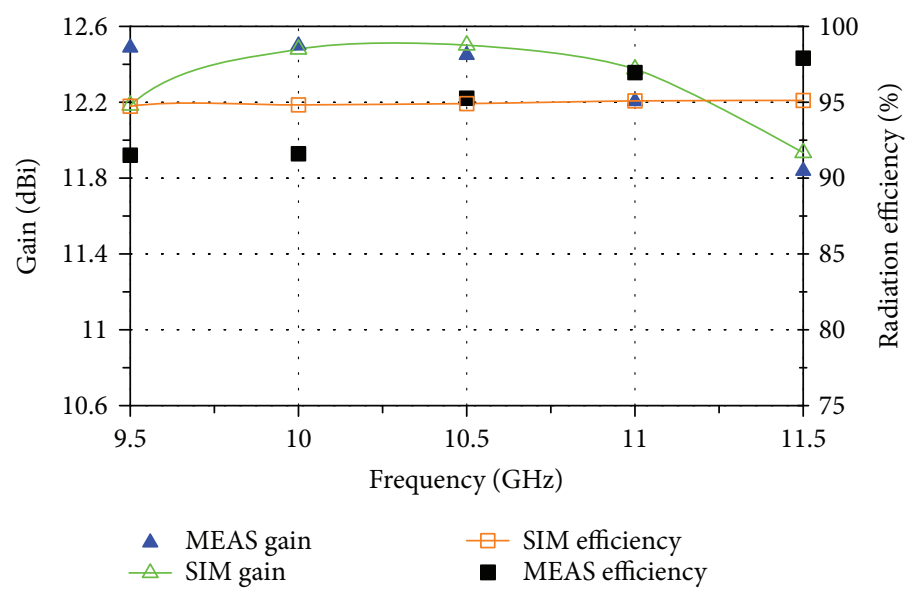

FIGURE 12: Maximum gain versus frequency of the proposed lens antenna.

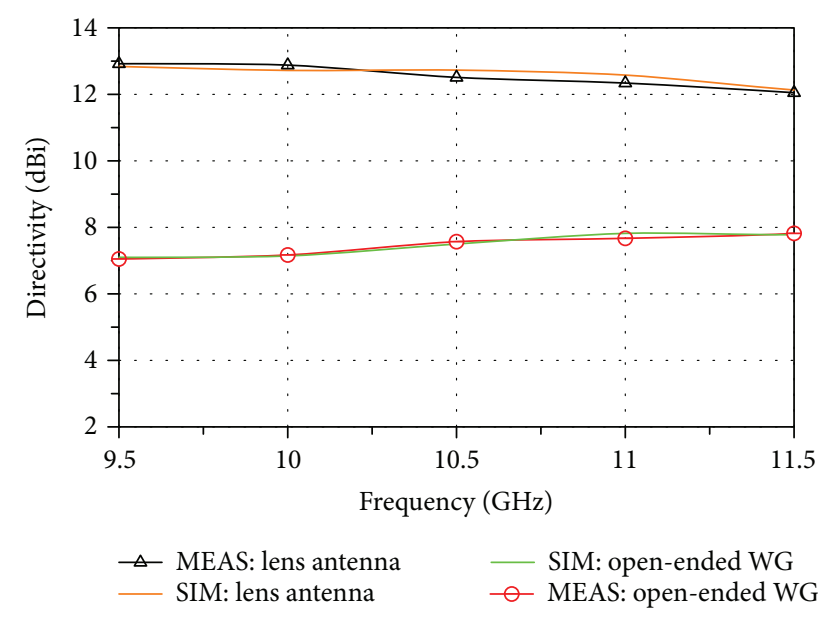

FIGURE 13: Comparison of the maximum directivity of the proposed lens and an open-ended waveguide.

\section{Conclusion}

In this paper, a new design for a metal lens formed by twelve metallic rings has been presented. The lens has been analyzed using characteristic modes in order to evaluate the performance of the currents on the metallic rings and provide a physical insight into the lens operation. An open-ended waveguide with a resonant ring is used as the primary feed for the metallic lens. The resulting structure improves the maximum directivity with respect to the feeder along a large bandwidth (more than $5 \mathrm{~dB}$ ) and provides a good crosspolar level (better than $-35 \mathrm{~dB}$ ).

\section{Conflicts of Interest}

The authors declare that they have no conflicts of interest.

\section{Acknowledgments}

This work has been supported by the Spanish Ministry of Economy and Competitiveness (Ministerio de Economía y
Competitividad) under the projects TEC2016-79700-C2-1$\mathrm{R}$ and TEC2016-78028-C3-3-P and college scholarship grant of the National University of Chimborazo.

\section{References}

[1] D. Sánchez-Escuderos, M. Cabedo-Fabrés, E. AntoninoDaviu, and M. Ferrando-Bataller, "Low-profile planar lens with multilevel FSS for directivity enhancement," in 2014 IEEE Antennas and Propagation Society International Symposium (APSURSI), pp. 2076-2077, Memphis, TN, USA, 2014.

[2] D. Sánchez-Escuderos, M. Cabedo-Fabrés, E. AntoninoDaviu, and M. Ferrando-Bataller, "Microwave planar lens antenna designed with a three-layer frequency-selective surface," IEEE Antennas and Wireless Propagation Letters, vol. 16, pp. 904-907, 2017.

[3] D. Sánchez-Escuderos, M. Cabedo-Fabrés, E. AntoninoDaviu, and M. Ferrando-Bataller, "Double-fed multilevel frequency selective surface for low-profile planar lens," in 2015 IEEE International Symposium on Antennas and Propagation \& USNC/URSI National Radio Science Meeting, pp. 989-990, Vancouver, BC, Canada, 2015.

[4] E. Plaza, G. Leon, S. Loredo, and F. Las-Heras, "A simple model for analyzing transmitarray lenses," IEEE Antennas and Propagation Magazine, vol. 57, no. 2, pp. 131-144, 2015.

[5] E. G. Plaza, G. Leon, S. Loredo et al., "An ultra-thin 2-bit near-field transmitarray lens," IEEE Antennas and Wireless Propagation Letters, vol. 16, pp. 1784-1787, 2017.

[6] S. V. Hum and J. Perruisseau-Carrier, "Reconfigurable reflectarrays and array lenses for dynamic antenna beam control: a review," IEEE Transactions on Antennas and Propagation, vol. 62, no. 1, pp. 183-198, 2014.

[7] Z. C. Hao, J. Wang, Q. Yuan, and W. Hong, "Development of a low-cost $\mathrm{THz}$ metallic lens antenna," IEEE Antennas and Wireless Propagation Letters, vol. 16, pp. 1751-1754, 2017.

[8] M. Jiang, Z. N. Chen, Y. Zhang, W. Hong, and X. Xuan, "Metamaterial-based thin planar lens antenna for spatial beamforming and multibeam massive MIMO," IEEE Transactions on Antennas and Propagation, vol. 65, no. 2, pp. 464-472, 2017.

[9] H. Li, G. Wang, J. Liang, X. Gao, H. Hou, and X. Jia, "Singlelayer focusing gradient metasurface for ultrathin planar lens antenna application," IEEE Transactions on Antennas and Propagation, vol. 65, no. 3, pp. 1452-1457, 2017. 
[10] R. Garbacz and R. Turpin, "A generalized expansion for radiated and scattered fields," IEEE Transactions on Antennas and Propagation, vol. 19, no. 3, pp. 348-358, 1971.

[11] R. F. Harrington and J. R. Mautz, "Theory of characteristic modes for conducting bodies," IEEE Transactions on Antennas and Propagation, vol. 19, no. 5, pp. 622-628, 1971.

[12] R. F. Harrington and J. R. Mautz, "Computation of characteristic modes for conducting bodies," IEEE Transactions on Antennas and Propagation, vol. 19, no. 5, pp. 629-639, 1971.

[13] M. Cabedo-Fabres, E. Antonino-Daviu, A. Valero-Nogueira, and M. Ferrando-Bataller, "The theory of characteristic modes revisited: a contribution to the design of antennas for modern applications," IEEE Antennas and Propagation Magazine, vol. 49, no. 5, pp. 52-68, 2007.

[14] A. Salih, Z. Chen, and K. Mouthaan, "Characteristic mode analysis and metasurface-based suppression of higher order modes of a $2 \times 2$ closely spaced phased array," IEEE Transactions on Antennas and Propagation, vol. 65, no. 3, pp. 11411150, 2017.

[15] Y. Chen and C.-F. Wang, "Electrically small UAV antenna design using characteristic modes," IEEE Transactions on Antennas and Propagation, vol. 62, no. 2, pp. 535-545, 2014.

[16] E. Antonino-Daviu, M. Cabedo-Fabres, M. Sonkki, N. Mohamed-Hicho, and M. Ferrando-Bataller, "Design guidelines for the excitation of characteristic modes in slotted planar structures," IEEE Transactions on Antennas and Propagation, vol. 64, no. 12, pp. 5020-5029, 2016.

[17] M. Capek, P. Hazdra, M. Masek, and V. Losenicky, "Analytical representation of characteristic mode decomposition," IEEE Transactions on Antennas and Propagation, vol. 65, no. 2, pp. 713-720, 2017.

[18] D. Santillán-Haro, E. Antonino-Daviu, D. Sánchez-Escuderos, and M. Ferrando-Bataller, "Single layer metasurface lens antenna," in 2017 11th European Conference on, Antennas and Propagation (EUCAP), IEEE, pp. 1134-1138, Paris, France, 2017.

[19] F. H. Lin and Z. N. Chen, "Low-profile wideband metasurface antennas using characteristic mode analysis," IEEE Transactions on Antennas and Propagation, vol. 65, no. 4, pp. 17061713, 2017.

[20] D. Santillán-Haro, E. Antonino-Daviu, D. Sánchez-Escuderos, and M. Ferrando-Bataller, "Metamaterial lens design using characteristics modes," in Numerical Electromagnetic and Multiphysics Modeling and Optimization for RF, Microwave, and Terahertz Applications (NEMO), 2017 IEEE MTT-S International Conference on, pp. 37-39, Sevilla, Spain, 2017.

[21] Dassault Systemes, CST Microwave Studio, Suite, 2017.

[22] “Altair, “FEKO”, Release,” 2017, http://www.feko.info. 


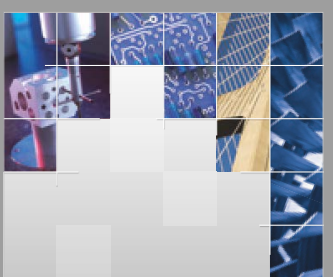

\section{Enfincering}
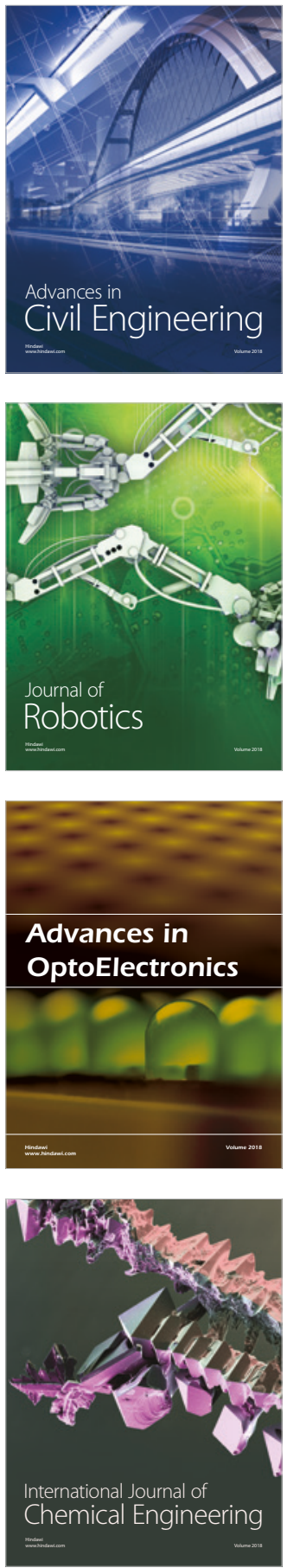

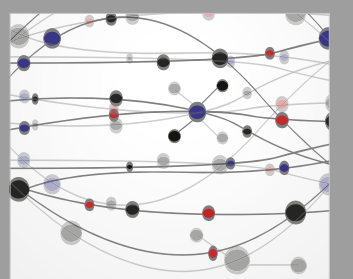

\section{Rotating \\ Machinery}

The Scientific World Journal

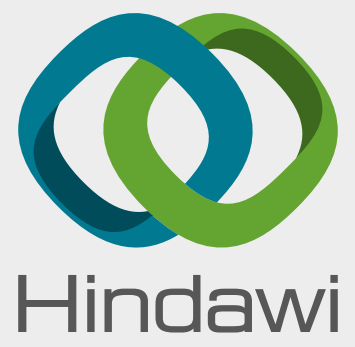

Submit your manuscripts at

www.hindawi.com
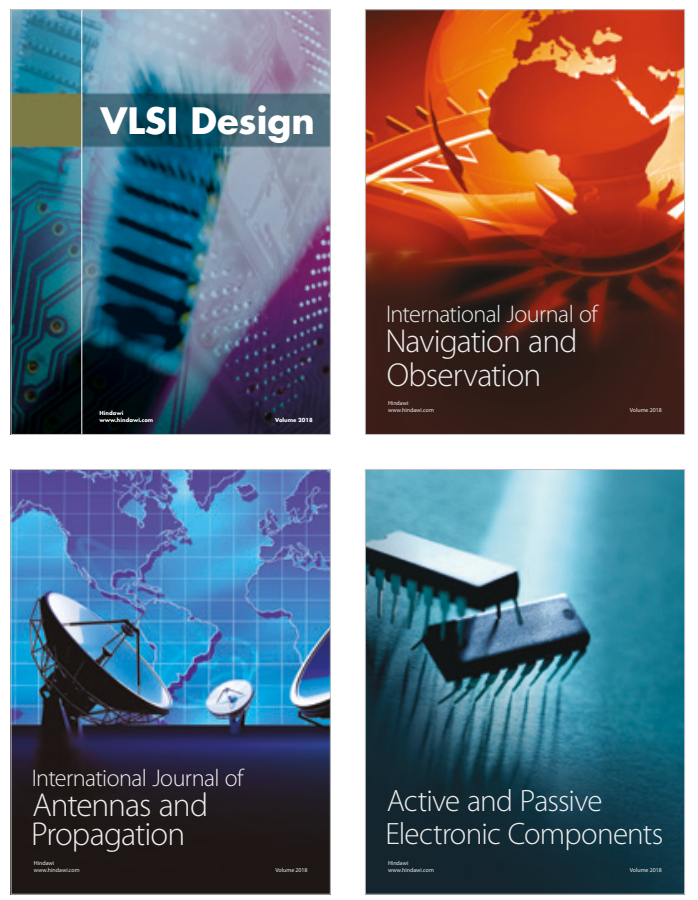
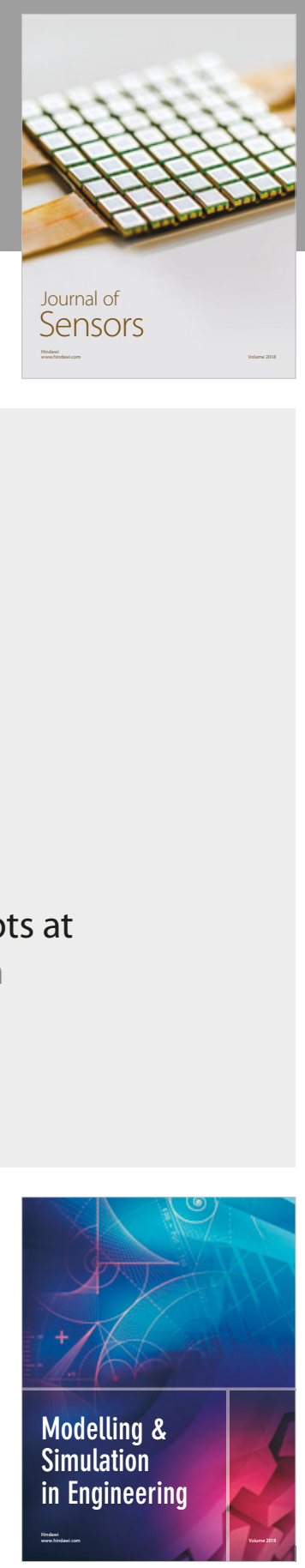

\section{Advances \\ Multimedia}
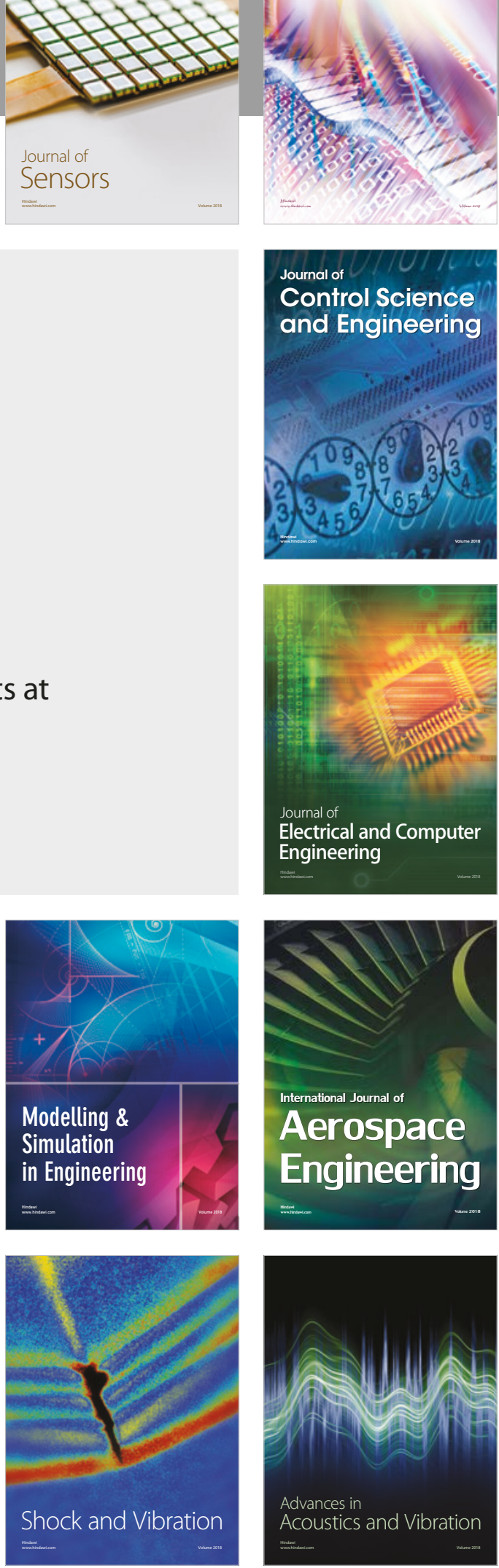